Int. J. Contemp. Math. Sci., Vol. 2, 2007, no. 17, 825 - 829

\title{
On Observability of Affine Control Systems on Lie Groups
}

\author{
Ayse Kara \\ Dept. of Maths., Yildiz Technical University \\ Davutpasa, 34210 Istanbul, Turkey \\ kara@yildiz.edu.tr
}

\begin{abstract}
In this work, observability of affine control systems on Lie groups is studied. With the distance preserving map as an output, distinguishability is preserved in order to have observability. Existence of distance preserving map on a simply connected 2nd degree nilpotent Lie group $G$ is satisfied and an example is given.
\end{abstract}

Mathematics Subject Classification: 93B07, 93B27

Keywords: observability, indistinguishability, distance preserving, affine algebra, semidirect product

\section{Introduction}

Let $G$ be a connected Lie group and $L(G)$ be its Lie algebra. The affine group $\operatorname{Af}(G)$ of $G$ is the semi-direct product of $\operatorname{Aut}(G)$ and $G, \operatorname{Af}(G)=\operatorname{Aut}(G) \times{ }_{s} G$. The group operation of $\operatorname{Af}(G)$ is

$$
\left(\phi, g_{1}\right) \cdot\left(\psi, g_{2}\right)=\left(\phi \circ \psi, g_{1} \phi\left(g_{2}\right)\right)
$$

If we denote by 1 the identity element of $\operatorname{Aut}(G)$ and by $e$ the identity element of $G$, then the group identity of $\operatorname{Af}(G)$ is $(1, e)$ and $\left(\phi^{-1}, \phi^{-1}\left(g^{-1}\right)\right)$ is the inverse of $(\phi, g) \in \operatorname{Af}(G)$. Therefore, $g \rightarrow(1, g)$ and $\phi \rightarrow(\phi, e)$ embed $G$ into $\operatorname{Af}(G)$ and $\operatorname{Aut}(G)$ into $\operatorname{Af}(G)$, respectively. Thus, $G$ and $\operatorname{Aut}(G)$ are closed subgroups of $\operatorname{Af}(G)$. There is a natural transitive action

$$
\operatorname{Af}(G) \times G \rightarrow G
$$

defined by

$$
\left(\phi, g_{1}\right) \cdot g_{2} \rightarrow g_{1} \phi\left(g_{2}\right)
$$


where $\left(\phi, g_{1}\right) \in \operatorname{Af}(G)$ and $g_{2} \in G$. Indeed, if it is taken $g_{2}=e$, then $\left(\phi, g_{1}\right) \cdot e=$ $g_{1}$ since $\phi\left(g_{2}\right)=e$.

The Lie algebra $\operatorname{af}(G)$ of $\operatorname{Af}(G)$ is the semi-direct product of aut $(G)$ and $L(G)$, where $\operatorname{aut}(G)$ is the Lie algebra of $\operatorname{Aut}(G)$. Lie bracket is given by

$$
\left[\left(D_{1}, X_{1}\right),\left(D_{2}, X_{2}\right)\right]=\left(\left[D_{1}, D_{2}\right], D_{1} X_{2}-D_{2} X_{1}+\left[X_{1}, X_{2}\right]\right) .
$$

An affine control system $\Sigma=(G, \mathcal{D}, p, V)$ on a Lie group $G \subset \operatorname{Af}(G)$ is determined by the family of differential equations :

$$
\begin{gathered}
\dot{x}=(D+X)(g)+\sum_{j=1}^{d} u_{j}(t)\left(D^{j}+Y^{j}\right)(g) \\
y=p(g) \in V,
\end{gathered}
$$

where $g \in G ; D, D^{1}, \ldots, D^{d} \in \operatorname{aut}(G)$ and $X, Y^{1}, \ldots, Y^{d} \in L(G) ; u \in U$, the class of unrestricted piecewise constant controls. The output space $V$ is a Lie group and in this work, we consider the output map

$$
p: G \rightarrow V
$$

is a distance preserving map.

Then, the dynamic is given by

$$
\mathcal{D}=\left\{D+X+\sum_{j=1}^{d} u_{j}\left(D^{j}+Y^{j}\right) \mid u \in \mathbb{R}^{d}\right\} .
$$

In general, affine control systems define richer class of systems than linear and bilinear classes. If the affine control system is considered on an abelian Lie group, then it becomes a linear control system. In fact, for the abelian Lie group case since any bracket between the elements of the Lie algebra is null the affine system turns to the form of the linear control system. If it is considered $X=0$ and $Y^{1}=Y^{2}=\ldots=Y^{d}=0$ for affine control system on a Lie group, then it becomes a bilinear control system.

\section{Observability}

Let $\Sigma=(G, \mathcal{D}, p, V)$ be an affine control system on Lie group $G \subset \operatorname{Af}(G)$. $\Sigma$ induces a group

$$
G_{\Sigma}=\left\{Z_{t_{1}}^{1} \circ Z_{t_{2}}^{2} \circ \ldots \circ Z_{t_{r}}^{r} \mid Z^{i} \in \mathcal{D}, t_{i} \in \mathbb{R}\right\}
$$

and a semi-group

$$
S_{\Sigma}=\left\{Z_{t_{1}}^{1} \circ Z_{t_{2}}^{2} \circ \ldots \circ Z_{t_{r}}^{r} \mid Z^{i} \in \mathcal{D}, t_{i} \geq 0\right\}
$$


of global diffoemorphisms on $G$.

$$
G_{\Sigma}(g)=\left\{\varphi(x) \mid \varphi \in G_{\Sigma}\right\}
$$

is the orbit of the system at $\mathrm{g}$. These orbits are the partitions of the state space and each of $G_{\Sigma}(g)$ at $g \in G$ has a differentiable manifold structure. Therefore, it is possible to restrict $\Sigma$ over its orbits.

Definition : Two elements $g_{1}, g_{2} \in G$ are indistinguishable by $\Sigma$, if

$$
p\left(\varphi\left(g_{1}\right)\right)=p\left(\varphi\left(g_{2}\right)\right), \forall \varphi \in S_{\Sigma}
$$

$\Sigma$ is said to be observable if there are no two points of $G$ which are indistinguishable by $\Sigma$.

Definition : A map $p:(G, \rho) \rightarrow(V, \sigma)$ is called distance preserving, if for any $g_{1}, g_{2} \in G$

$$
\sigma\left(p\left(\left(g_{1}\right), p\left(g_{2}\right)\right)=\rho\left(g_{1}, g_{2}\right) .\right.
$$

A distance preserving mapping is automatically injective.

Because of the special form of the solution it is convenient to study the observability problem on $\Sigma=(G, D+X, p, V)$, independent of controlled vectors.

Therefore, two elements $g_{1}, g_{2} \in G$ are indistinguishable, if

$$
p\left((D+X)_{t}\left(g_{1}\right)\right)=p\left((D+X)_{t}\left(g_{2}\right)\right), \forall t \geq 0
$$

where $(D+X)_{t}$ is the one-parameter group of authomorphisms which is induced by $D+X \in a f(G)$.

Theorem 1 : If $\Sigma=(G, D+X, p, V)$ is a control system on $G$ with a distance preserving out put map $\mathrm{p}$, then this system is observable.

Proof : Consider $p:(G, \rho) \rightarrow(V, \sigma)$. If we take any two distinct elements $g_{1}$ and $g_{2}$ of $G$, then $\rho\left(g_{1}, g_{2}\right)>0$. Therefore, if $\rho\left(\varphi\left(g_{1}\right), \varphi\left(g_{2}\right)\right)>0$, then we

$$
\sigma\left(p\left(\varphi\left(g_{1}\right)\right), p\left(\varphi\left(g_{2}\right)\right)\right)=\rho\left(g_{1}, g_{2}\right)>0
$$

Thus, this system is observable.

Definition : A Lie algebra $L(G)$ is called 2nd degree nilpotent, if

$$
L(G)^{1}=L(G), L(G)^{2}=[L(G), L(G)] \text { and } L(G)^{3}=\left[L(G)^{2}, L(G)\right]=0 .
$$

Theorem 2 : If $\Sigma=(G, D+X, p, V)$ is a control system on a simply connected 2nd degree nilpotent Lie group $G$, then there exists a distance preserving mapping of $G$ to a Lie group $V$.

Proof : The usual metric on $\mathbb{R}^{n}$ extends a distance preserving metric on simply connected 2 nd degree nilpotent Lie group $G$. 


\section{Example}

Let $G$ be the Heisenberg group of dimension 3,

$$
G=\left\{g=\left(\begin{array}{ccc}
1 & a & c \\
0 & 1 & b \\
0 & 0 & 1
\end{array}\right) \mid a, b, c \in \mathbb{R}\right\}
$$

The Lie algebra of $G$ is given by

$$
L(G)=\operatorname{Span}_{\mathcal{L} . \mathcal{A} .}\left\{Y_{1}=\left(\begin{array}{lll}
0 & 1 & 0 \\
0 & 0 & 0 \\
0 & 0 & 0
\end{array}\right), Y_{2}=\left(\begin{array}{lll}
0 & 0 & 0 \\
0 & 0 & 1 \\
0 & 0 & 0
\end{array}\right)\right\} \text {, where }\left[Y_{1}, Y_{2}\right]=Y_{3} .
$$

Heisenberg Lie group is topologically diffeomophic to $\mathbb{R}^{3}$ via

$$
\psi:\left(\begin{array}{ccc}
1 & a & c \\
0 & 1 & b \\
0 & 0 & 1
\end{array}\right) \rightarrow(a, b, c)
$$

If we consider the usual metric $\rho=\left(\sum_{i=1}^{3}\left|x_{i}-y_{i}\right|\right)^{1 / 2}$ on $\mathbb{R}^{3}$, then $\psi^{-1}(\rho)$ defines a metric on $G$. If we take the affine control system $\Sigma$ on $G$ which is given with the dynamic

$$
\begin{gathered}
\mathcal{D}=\left\{\left(\begin{array}{lll}
1 & 0 & 0 \\
0 & 0 & 0 \\
0 & 0 & 1
\end{array}\right)+u\left(\begin{array}{lll}
0 & 0 & 0 \\
0 & 0 & 1 \\
0 & 0 & 0
\end{array}\right) \mid u \in U,\right. \\
\left.\left(\begin{array}{lll}
1 & 0 & 0 \\
0 & 0 & 0 \\
0 & 0 & 1
\end{array}\right) \in \operatorname{Der}(L(G)) \text { and }\left(\begin{array}{lll}
0 & 0 & 0 \\
0 & 0 & 1 \\
0 & 0 & 0
\end{array}\right) \in L(G)\right\} .
\end{gathered}
$$

Here,

$\operatorname{Der}(L(G))=\{D \in \operatorname{End}(L(G)) \mid D[X, Y]=[D(X), Y]+[X, D(Y)], \forall X, Y \in L(G)\}$

Existence of a distance preserving map permits that any two different solutions in $G$ has disjoint outputs in $V$.

\section{References}

[1] V. Ayala, A. H. Kara and E. Kizil, Observability of General Linear Pairs, Computers and Mathematics with Applications, Vol.39 (1-2), (2000), 3543. 
[2] A. Isidori, Nonlinear Control Systems : an introduction, Springer Verlag, 1989.

[3] V. Jurdjevic, Geometric Control Theory, Cambridge University Press, 1997.

[4] A. Kara A. and L. A. B. San Martin, Controllability of Affine Control System for the Generalized Heisenberg Lie Groups, International Journal of Pure and Applied Mathematics, Vol.29, No.1, (2006), 1-6.

Received: December 11, 2006 